\title{
Avaliação da trajetória, no SUS, de pacientes atendidos na clínica cirúrgica de um serviço de emergência a partir de seus diagnósticos histopatológicos
}

\author{
Evaluation of patient's rout aided on surgical clinic of an \\ emergency service on SUS after their histopathologic diagnostics
}

\author{
Maria Luisa Duarte ${ }^{1}$ \\ Victor Hugo Farias Costa ${ }^{2}$ \\ Lívia de Souza Mota ${ }^{2}$
}

\section{Resumo}

Assim como na maioria dos serviços de emergência do Brasil, a Unidade de Emergência Dr. Armando Lages, principal hospital com este perfil de Alagoas, tem seu potencial limitado pelas lacunas no planejamento e na execução da Atenção Básica. A rede básica de atenção à saúde deveria constituir a principal porta de entrada no sistema. Afirma-se que na Atenção Básica 80\% dos problemas de saúde da população sejam resolvidos. O que se verifica, contudo, é o escamoteamento de suas atribuições e potencialidades. Define-se que a Saúde da Família (SF) deve constituir equipes de saúde formadas por médicos, enfermeiros, auxiliares de enfermagem e agentes comunitários, em trabalho interdisciplinar com a definição de um território de abrangência. O processo de referência e contra-referência é imprescindível para que haja uma continuidade das ações e compete ao serviço municipal definir, no âmbito municipal ou regional, os serviços disponíveis para os níveis de maior complexidade. A SF deverá, portanto, em condições especiais, indicar o encaminhamento para consultas especializadas, assim como para os serviços de diagnóstico e internações hospitalares. Na prática, porém, os serviços de atenção terciária acabam absorvendo a demanda proveniente de problemas na rede básica. É o caso da Unidade de Emergência Dr. Armando Lages, na qual, mensalmente, a taxa de ocupação dos leitos é de 109,34\%, absorvendo pacientes que não encontram outra referência para solucionar seus problemas de saúde. Obedecendo às normas que regem a Pesquisa em Seres Humanos, foi desenvolvido um estudo descritivo retrospectivo e prospectivo, aplicando-se questionário de perguntas estruturadas a pacientes da clínica cirúrgica da Unidade de Emergência Dr. Armando Lages que tiveram diagnósticos histopatológicos das peças cirúrgicas efetuados no período de janeiro de 2004 a dezembro de 2006. A análise dos dados revelou que, na trajetória dos pacientes pelo Sistema Único de Saúde, princípios doutrinários e organizativos do SUS estão desordenados, confirmando a hipótese do estudo, de que o número evidente de diagnósticos histopatológicos de neoplasias malignas e doenças infecto-parasitárias sugere falhas no planejamento em saúde da rede de Atenção Primária á Saúde.

Palavras-chave: Diagnóstico; Planejamento em Saúde; Serviços Médicos de Emergência; Sistema Único de Saúde (SUS).
Key Words: Health Care Costs; Diagnosis; Health Planning; Emergency Medical Services; Single Health System.

${ }^{1}$ Doutora em Patologia pela UFF. Professora adjunta, Departamento de Patologia, Universidade Estadual de Ciências da Saúde de Alagoas, Maceió, Alagoas, Brasil.

${ }^{2}$ Acadêmicos do quinto ano de Medicina. Universidade Estadual de Ciências da Saúde de Alagoas, Maceió, Alagoas, Brasil. 


\begin{abstract}
Like most of the emergency services in Brazil, Dr. Armando
\end{abstract} Lages Emergency Unit, the main kind of hospital on its profile has its potential limited due to planning and execution weaknesses in basic bealthcare. The basic healthcare network should be the health system's main point.. It is stated that in basic care, $80 \%$ of the population's health problems should be resolved. However, in real situation we can see a retraction in its attributions and potential. The definition of the Family Health Program (Brazilian acronym - PSF) states that there must be composed by health teams formed by doctors, nurses, nursing assistants and communitarian representatives composing an interdisciplinary working environment. The team must work within a defined area of coverage. The reference and contra-reference process is indispensable to enable the continuity of activities and the municipal service must define the available services for the most complex levels on a municipal and regional basis. The Family Health Program must therefore, coordinate appointments with specialists, diagnosis services and hospital admissions. Actually, the practical routine, however, the tertiary care services end up absorbing the demand originating from the shortcomings in the basic network. This is the case at Dr. Armando Lages Emergency Unit, where monthly the bed occupation rate is $109.34 \%$, as patients who could not find other means to solve their health problems are absorbed. A retrospective and prospective descriptive study was developed following the rules which conduct research in Human Beings, by applying a questionnaire of structured questions to patients in the surgical clinic at the Dr. Armando Lages Emergency Unit who had bystopatological diagnosises of surgical or biopsy parts, carried out in the period between January 2004 to December 2006. The data analysis revealed that in the trajectory of patients in the National Health System (SUS), the doctrinaire and organizational principles of SUS are disorderly, confirming in the case under study, that the evident number of hystopatological diagnosises of malign neoplasias and infected-parasitic diseases suggest that there are shortcomings in the bealth planning of the primary care network.

\section{Introdução}

O primeiro serviço de pronto-socorro de Alagoas surgiu em 1936, por ocasião da ampliação da Santa Casa de Misericórdia de Maceió; a instituição manteve esse serviço até o ano de 1955.

Em 1956, a prefeitura municipal de Maceió assumiu a responsabilidade por este serviço, que passou a ser denominado Serviço Municipal de Pronto-Socorro. No entanto, apenas em 1979 o hospital adquiriu sede própria, passando a denominar-se Unidade de Emergência Dr. Armando Lages, pelo Decreto Municipal n. ${ }^{\circ}$ 1.676, vinculado à Fundação Governador Lamenha Filho (FUNGLAF).

O hospital tem como missão "prestar assistência médico-hospitalar de urgência e emergência, através de equipe multidisciplinar, de forma eficaz à população". No entanto, assim como na maioria dos serviços de emergência do Brasil, a Unidade de Emergência Dr. Armando Lages tem seu potencial limitado pelas lacunas no planejamento e na execução da Atenção Básica.

A rede básica de atenção à saúde deveria constituir a principal porta de entrada no sistema de saúde. Aliás, espera-se dela muito mais que isso. Afirma-se que, na Atenção Básica, 80\% dos problemas de saúde da população sejam resolvidos ${ }^{1}$. O que se verifica, contudo, é o escamoteamento de suas atribuições, consagradas em 1978, na Conferência Mundial de Saúde em Alma Ata, e de sua importância fundamental na efetivação do SUS ${ }^{2}$.

Este problema está diretamente vinculado a um dos principais entraves à implementação do SUS: a adoção de um modelo tecno-assistencial mais resolutivo e adequado a cada município ${ }^{1,2}$.

Este modelo sempre se mostrou incapaz de dar conta, do ponto de vista epidemiológico e econômico, dos problemas de saúde e de modificar o modo de nascer, viver, adoecer e morrer das pessoas, bem como de controlar as doenças endêmicas ${ }^{3}$.

A existência de populações vulneráveis levou o Ministério da Saúde, a partir da década de 1970, a repensar o modelo de atenção à saúde (clínico-individual), incluindo as ações programáticas de saúde, principalmente para combater doenças endêmicas, como hanseníase e tuberculose; combater doenças sexualmente transmissíveis ${ }^{2}$. Essas ações propõem, no caso dos programas de combate às doenças, 
o atendimento das pessoas acometidas, mas apresenta uma enorme incapacidade em definir e operacionalizar ações de promoção em saúde. Este modelo ficou conhecido como modelo de Ações Programáticas.

Somente após a VIII Conferência Nacional de Saúde, em Brasília, em 1986, houve uma mudança no sistema de saúde, que passou então para um Sistema Único e Descentralizado de Saúde (SUDS), a partir do decreto de 20 de julho de 1987 . Nesta ocasião, debateu-se a saúde como direito social, e a Atenção Básica como estratégia para a mudança do perfil epidemiológico do país ${ }^{4}$.

Diante deste novo contexto, surge, em 1988, por meio da Constituição Federal, um modelo de saúde que representa a inflexão da evolução institucional do Brasil. Foi determinada uma total reorganização institucional, ao introduzir um novo conceito jurídico no campo das políticas públicas de saúde - eis, então, o Sistema Único de Saúde (SUS), modelo assistencial em vigor e que tem como princípio central a saúde como direito universal ${ }^{5}$.

Por volta de 1991, dá-se início ao processo de descentralização do SUS, com a criação do Programa de Agentes Comunitários de Saúde (PACS), gerando um novo ordenamento nos serviços de saúde a nível ambulatorial e domiciliar ${ }^{4}$.

Já em 1994, o Ministério da Saúde, consciente de que a estrutura clássica das unidades básicas de saúde não vinha apresentando satisfatório poder de resolução, decide, a partir de experiências positivas nas regiões Norte e Nordeste, implantarem o Programa de Saúde da Família (PSF), incorporando e ampliando a atuação do PACS. Com essa iniciativa, o Ministério da Saúde ambicionou a reorganização da prática de atenção à saúde ${ }^{2,4}$.

Define-se que o PSF deve constituir equipes de saúde formadas por médicos, enfermeiros, auxiliares de enfermagem e agentes comunitários, em trabalho interdisciplinar. A equipe deve trabalhar com a definição de um território de abrangência. Recomenda-se que cada equipe responsabilize-se por uma área com 600 a 1.000 famílias, com limite máximo de 4.500 habitantes. As equipes deverão fazer o cadastro das famílias por meio das visitas domiciliares ${ }^{6,7}$.

O processo de referência e contrareferência é imprescindível para que haja uma continuidade das ações, e compete ao serviço municipal definir, no âmbito municipal ou regional, os serviços disponíveis para os níveis de maior complexidade. O PSF deverá, portanto, em condições especiais, indicar o encaminhamento para consultas especializadas, assim como para os serviços de diagnóstico e internações hospitalares?

Na prática, porém, os serviços de atenção terciária acabam absorvendo a demanda proveniente de falhas na rede básica. É o caso da Unidade de emergência Dr. Armando Lages, na qual, mensalmente, a taxa de ocupação dos leitos é de 109,34\%, absorvendo pacientes que não encontram outra referência para solucionar seus problemas de saúde.

Os serviços de emergência são serviços de saúde extensamente estudados em diversos países há vários anos. Esses trabalhos objetivam um conhecimento mais profundo das nuances de cada serviço, para que sua estruturação seja feita de forma mais racional ${ }^{8,9}$. Assim, é importante que cada serviço seja estudado dentro de seu contexto particular, para que as decisões possam ser tomadas com o máximo de adequação.

A despeito do reconhecimento da importância de se estudar as emergências, curiosamente elas têm sido objeto pouco frequente de pesquisas no Brasil. Frente a isso, serviços como a Unidade de Emergência Dr. Armando Lages terminam sendo porta de entrada de um sistema desintegrado e com uma frágil estrutura hierárquica.

\section{Casuística e Método}

Foi desenvolvido um estudo descritivo retrospectivo, aplicando-se questionário de perguntas estruturadas a pacientes da clínica cirúrgica da Unidade de Emergência Dr. Armando Lages que tiveram diagnósticos histopatológicos das peças cirúrgicas ou biópsia efetuados no período de janeiro de 2004 a dezembro de 2006. A amostragem incluiu também suas respectivas notificações colhidas dos prontuários e das fichas de atendimento naquele hospital. 
Obedecendo às normas que regem a Pesquisa em Seres Humanos, a Declaração de Helsinki de 1964 e a Resolução no 196/96 do Ministério da Saúde, o projeto desta pesquisa foi encaminhado e submetido ao Comitê de Ética em Pesquisa da Universidade Estadual de Ciências da Saúde de Alagoas - UNCISAL -, para devida aprovação, antes de ser iniciado.

\section{Resultados}

Dos 54 casos escolhidos, 52 (96,29\%) foram incluídos no presente estudo, visto que dois $(3,70 \%)$ faleceram.
Quanto à procedência, 38 pacientes ( $95 \%)$ eram da capital alagoana e dois (5\%), de outros municípios. Os diagnósticos histopatológicos firmados e suas respectivas frequências estão ilustrados no gráfico 1.

Constatou-se que $75 \%$ dos pacientes relataram não haver qualquer ação de prevenção e promoção à saúde desenvolvidas no seu bairro ou proximidades, $15 \%$ que visitas domiciliares são realizadas com este fim, $5 \%$ que palestras são oferecidas e, outros $5 \%$ responderam que campanhas são feitas para esta finalidade, como no gráfico 2.

Perguntados se já haviam recebido a equipe de

Gráfico 1: Diagnósticos

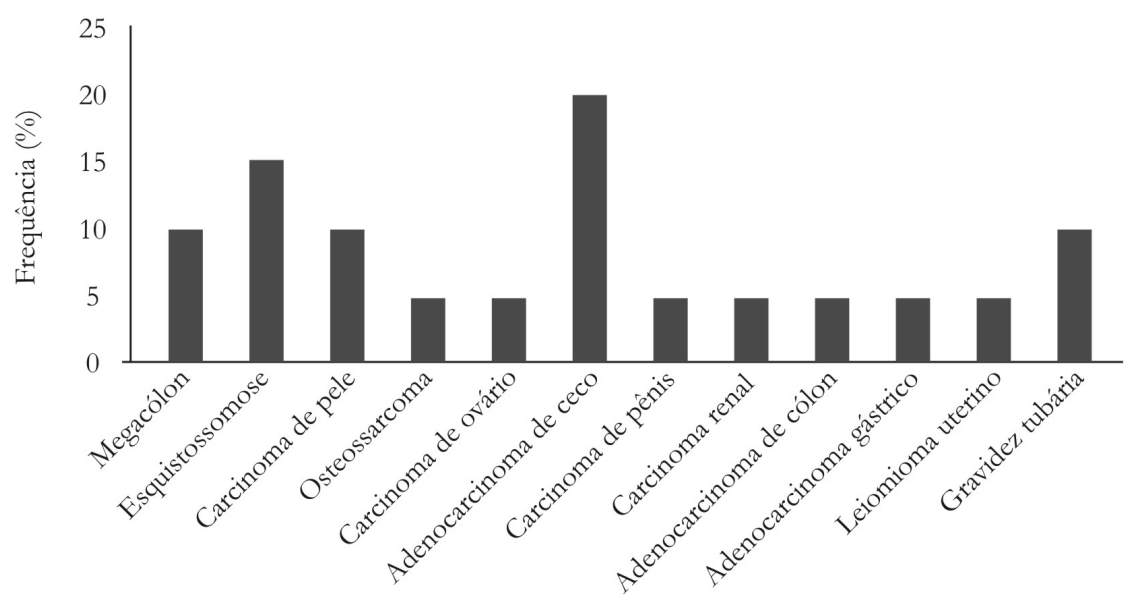

Gráfico 2: Ações de prevenção e promoção à saúde

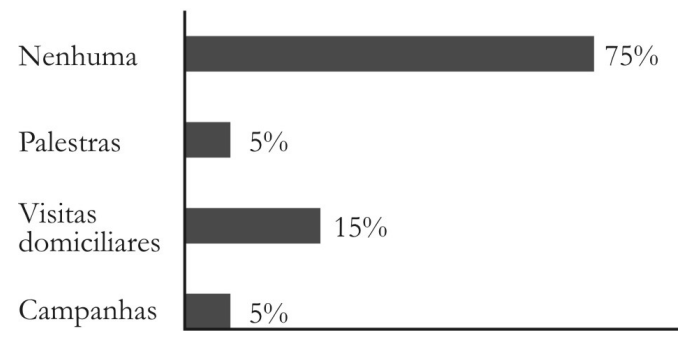


Saúde da Família em suas residências, 60\% afirmaram que sim, $40 \%$, que não. Ninguém relatou não haver equipe de Saúde da Família em seu bairro ou proximidades; o cadastramento das famílias por agentes comunitários de saúde foi realizado em $75 \%$ dos casos estudados.

Antes de recorrer à Unidade de Emergência Dr. Armando Lages, 10\% dos pacientes estudados buscaram outro serviço de saúde - dentre os serviços solicitados, $75 \%$ recorreram à minipronto-socorros, e $25 \%$, à Centros de Saúde. A grande maioria, 90\%, no entanto, recorreu imediatamente à Unidade de Emergência Dr. Armando Lages.

Dentre as razões que motivaram a busca pela U. E. Dr. Armando Lages, 70\% dos pacientes responderam que procuraram este serviço porque avaliaram seu problema como grave, $10 \%$ porque foram orientados pelo médico, $10 \%$ porque acreditavam que o médico da Unidade Básica de Saúde do seu bairro não poderia resolver seu problema, e $10 \%$ porque não conseguiram atendimento na Unidade Básica de Saúde de seu bairro, como mostra o gráfico 3.

Durante o tempo em que ficaram internados na Unidade de Emergência Dr. Armando Lages, 15\% dos pacientes entrevistados afirmaram ter havido intercorrências, $75 \%$ negaram tal fato. As intercorrências citadas foram: infecção da ferida cirúrgica $(66,6 \%)$ e infecção respiratória $(33,3 \%)$.

Gráfico 3: Razões que motivaram a procura pela U.E Dr. Armando Lages

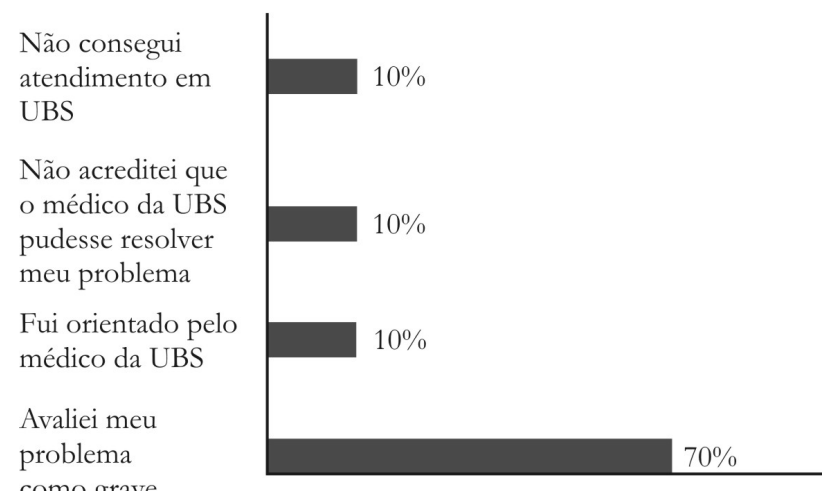

Quando perguntados se estavam sendo acompanhados por algum serviço de saúde, $60 \%$ dos entrevistados responderam que não estavam recebendo acompanhamento algum, e $40 \%$ relataram que sim. Destes, $62,5 \%$ disseram estavam sendo acompanhados pelo Hospital Universitário da Universidade Federal de Alagoas, 25\% responderam que estão sendo acompanhados pela Santa Casa de Misericórdia de Maceió, e 12,5\%, que estavam recebendo acompanhamento da Equipe de PSF de seu bairro/proximidades, como mostra o gráfico 4.

\section{Discussão}

A análise dos dados revelou que, na trajetória dos pacientes pelo Sistema Único de Saúde, princípios doutrinários e organizativos do SUS estão desordenados, confirmando a hipótese do estudo, de que o número evidente de diagnósticos histopatológicos de neoplasias malignas e doenças infecto-parasitárias sugere falhas no planejamento em saúde da rede de Atenção Primária à Saúde.

Mais precisamente, encontramos problemas na implementação dos princípios da integralidade, regionalização e hierarquização.

O princípio da integralidade é entendido como a abordagem da pessoa em sua totalidade, atendendo a todas as suas necessidades. Para isso, é importante a integração de ações, incluindo a promoção de saúde, prevenção de fazem acompanhamento

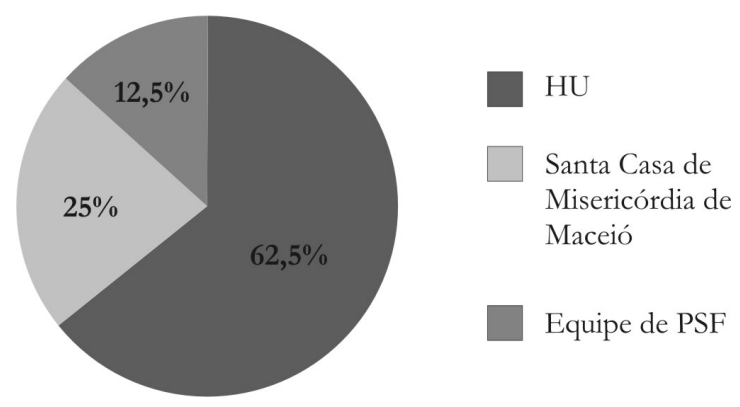


doenças, o tratamento e a reabilitação ${ }^{10}$. Uma vez que $75 \%$ dos pacientes relataram não haver qualquer ação de prevenção ou promoção de saúde nos seus bairros ou proximidades, fica claro o descumprimento deste princípio. Ao mesmo tempo, a integralidade pressupõe a articulação entre as várias políticas sociais, como forma de assegurar uma ação intersetorial aos indivíduos. O resultado que encontramos, de $60 \%$ dos pacientes que relataram não estarem sendo acompanhados por qualquer serviço de saúde, evidencia que o princípio da integralidade também não é cumprido neste sentido.

A Estratégia de Saúde da Família, concebida com o objetivo de reorientar o planejamento em saúde e a assistência à população, passando a ter como núcleo de cuidado as famílias e a comunidade, sendo as mesmas abordadas e compreendidas a partir do meio onde vivem, não está executando seus objetivos precípuos, de acordo com os resultados do estudo?.

O modelo SF, em seus anos de existência, tem encontrado grandes dificuldades na implementação de uma nova ética social e cultural, para concretizar o ideário de promoção de saúde. Sua sustentabilidade requer o estabelecimento contínuo de parcerias intersetoriais, articulando ações interdisciplinares de assistência, prevenção e promoção de saúde, de referenciamento e contrareferenciamento de pacientes $^{6,7}$.

Segundo a análise dos achados, a SF local não tem demonstrado poder de induzir mudanças significativas na organização do conjunto da rede, a ponto de assegurar à sua clientela acesso aos níveis de maior complexidade, nem parece caminhar para a universalização na cobertura, mesmo de ações básicas.

A regionalização e a hierarquização de serviços significa que os serviços devem ser organizados em níveis crescentes de complexidade, com definição e conhecimento da clientela a ser atendida. A regionalização é, na maioria das vezes, um processo de articulação entre os serviços já existentes $^{10}$. A hierarquização deve, além de proceder a divisão de níveis de atenção, garantir formas de acesso a serviços que componham toda a complexidade requerida para o caso, no limite de recursos disponíveis em uma dada região $0^{10,11}$.

A grande maioria dos pacientes estudados recorreu imediatamente à U. E. Dr. Armando Lages, sem antes ter buscado auxílio em outro serviço. As características de organização do processo de trabalho em serviços de emergência, isto é, a realização rápida do diagnóstico clínico e de disponibilidade imediata e ininterrupta de procedimentos diagnósticos e tarapêuticos justificam a sua procura direta pela maior parte da população, descaracterizando-os do seu principal objetivo, comprometendo a eficácia técnica para a consecução dos sistemas de urgência e emergência ${ }^{12,13}$.

Os fatores determinantes na decisão de se procurar a U. E. Dr. Armando Lages como primeira alternativa de atendimento mostram a fragilidade do sistema de referência e contrareferência - apenas 10\% dos pacientes responderam que foram orientados pelo médico a fazê-lo.

A Norma Operacional de Assistência à Saúde (NOAS SUS 01/01) é o instrumento de que deve lançar mão o gestor de saúde na implementação da regionalização e hierarquização dos serviços de saúde. Para garantir esse objetivo, a NOAS define a regionalização como macroestratégia de reorganização assistencial: "A macroestratégia de regionalização deverá contemplar uma lógica de planejamento integrado de maneira a conformar sistemas funcionais de saúde, ou seja, redes articuladas e cooperativas de atenção, referidas a territórios delimitados e a populações definidas, dotadas de mecanismos de comunicação e fluxos de interelacionamento que garantam o acesso dos usuários às ações e aos serviços de níveis de complexidade necessários para a resolução dos seus problemas de saúde, otimizando os recursos disponíveis"

No sistema de saúde, o atendimento às urgências e emergências é referenciado a partir do serviço de saúde em que o paciente recebeu o primeiro atendimento, entretanto, como o atendimento da demanda espontânea não se encontra regionalizado, a população se desloca desordenadamente. É a distorção do fluxo de atendimento no que se refere à regionalização da assistência à saúde ${ }^{12,13}$.

Dentre os pacientes que relataram estarem sendo 
acompanhados por algum serviço de saúde, $62,5 \%$ responderam que o Hospital Universitário da Universidade Federal de Alagoas realizava tal assistência. A explicação provável para essa constatação envolve uma série de fatores que interagem entre si, destacando-se o padrão de consumo de serviços de saúde pelos usuários e o posicionamento do H. U. no sistema de saúde. Além disso, o caráter da instituição, um hospital universitário, oferecendo recursos humanos altamente especializados e tecnologia de ponta no estado, constantemente divulgada por meios de comunicação, desempenha papel relevante no resultado encontrado ${ }^{14}$.

O percentual encontrado de acompanhamento dos pacientes pela Santa Casa de Misericórdia de Maceió, maior do que o encontrado pelas Unidades de Saúde da Família, suscita uma preocupação com a efetiva mudança do modelo assistencial proposto pelos princípios do SUS. O modelo médico-assistencial privatista é centrado no atendimento de doentes (demanda espontânea ou induzida pela oferta), com ênfase na assistência de alto custo, prestada principalmente pela rede contratada e conveniada com o SUS, apresentando sérios limites para uma atenção comprometida com a efetividade, equidade e necessidade de saúde, ainda que possa proporcionar uma assistência de qualidade em algumas situações.

\section{Conclusão}

O estudo mostra que existem barreiras ao acesso integral aos serviços de saúde, não só inerentes às condições dos usuários, mas também ao sistema de saúde. Alguns fatores envolvidos na organização dos serviços de saúde, como capacitação profissional para prevenção e promoção de saúde, falta de comprometimento dos profissionais atuantes no nível primário de atenção, falta de profissionais especializados e de exames subsidiários e outros procedimentos eletivos comprometem o trânsito eficiente dos pacientes por serviços de saúde resolutivos e organizados em níveis crescentes de complexidade. Soma-se a isso a falta de articulação entre todos os níveis de assistência, do primário à alta complexidade, impedindo a integralidade da atenção à saúde ${ }^{14}$.
Assim, o serviço de emergência acaba acolhendo esta população "perdida" na rede, portadora de consequências clínicas da ineficácia ou inexistência de ações de prevenção e promoção de saúde, atribuições regulamentarmente instituídas da rede de Atenção Básica.

A racionalidade do modelo de oferta de serviços de saúde deve ser voltada para as necessidades reais da população, atendendo à lógica do SUS: cada um dos níveis assistenciais deve estar capacitado a ser responsável pela atenção a uma determinada parcela da população, permitindo um conhecimento maior dos seus problemas de saúde em uma área delimitada, favorecendo todas as ações em uma forma integralizada de assistência à saúde. Os serviços devem ser organizados para que se possa acolher a clientela de acordo com a severidade do quadro e a necessidade requerida de recursos, para que esta população não se dirija aos pronto-socorros já apresentando complicações ou agravos francamente passíveis de prevenção pela rede de Atenção Básica ${ }^{13}$.

Diante da complexidade dos fatores associados à trajetória tortuosa e penosa dos pacientes estudados, fica claro que há um longo caminho a ser percorrido na reorganização do sistema de saúde. Um grande desafio é desenvolver uma política que garanta o uso apropriado dos serviços de emergência, a sua articulação com os demais componentes da rede pública de saúde e, por fim, o alinhamento final de todos os setores do sistema voltados a uma direção única: a qualidade da assistência à saúde.

\section{Referências}

1. Barakat SF. Caracterização da demanda do Serviço de Emergências Clínicas de um hospital terciário do município de São Paulo. [Dissertação]. São Paulo, Faculdade de Medicina, Universidade de São Paulo; 2004.

2. Barros AJ. Avaliação da cobertura do Programa Saúde da Família sob a atenção básica em município do Nordeste. Revista Brasileira de Saúde Materno Infantil. 2002;(2):12-25. 3. Brasil. Ministério da Saúde. Norma Operacional de Assistência à Saúde -SUS 01/2001. Brasília (DF): Ministério da Saúde; [s.d.]. 
4. Brasil. Ministério da Saúde. Relatório final da VIII

Conferência Nacional de Saúde. Brasília (DF): Ministério da Saúde; 1986.

5. Brasil. Senado Federal. Constituição 1988. Constituição

da República Federativa do Brasil. Brasília (DF): Presidência da República; 1988.

6. Campos FE. Cadernos de Saúde. Belo Horizonte: Coopmed; 1998. 109p.

7. Costa EM. Saúde da Família - uma abordagem interdisciplinar. Rio de Janeiro: Rubio; 2004. 75p.

8. Filho AJ. Serviço de Emergência em clínica médica do hospital universitário da UFSC: estudo do perfil da demanda. Arquivos Catarinenses de Medicina. 1990; (19): 37-44.

9. Furtado BM. O perfil da emergência do Hospital da Restauração: uma análise dos possíveis impactos após a municipalização dos serviços de saúde. Revista Brasileira de Epidemiologia. 2004; (7): 279-289.

10. Machado MF. Integralidade, formação de saúde, educação em saúde e as propostas do SUS - uma revisão conceitual. Ciência e saúde coletiva. 2007; (2): 14-29.

11. Salla J. Perfil da demanda de um serviço de ProntoSocorro. Saúde. 1989; (2): 71-80.

12. Sanchez J. Perfil de las demandas de atención médica en el servicio de urgência. Cirurgía y Cirujanos. 1998; (5): 21-39.

13. Santos NR. Desenvolvimento do SUS, rumos estratégicos e estratégias para visualização dos rumos. Ciência e Saúde Coletiva. 2006; (1): 9-35.

14. Teixeira CF. Promoção e vigilância da saúde no contexto da regionalização da assistência à saúde no SUS. Cadernos de Saúde Pública. 2002; (9):53-62.

Instituição de Fomento desta pesquisa:

FAPEAL - Fundação de Amparo à Pesquisa do Estado de Alagoas

$\mathrm{N}^{\circ}$ de cadastro: 2002.11.023-5

\section{Endereço para correspondência:}

Conj. Jardim Petrópolis II-E, $\mathrm{n}^{\circ} 55$

Maceió - AL

CEP 57.063-210

\section{Endereço eletrônico:}

maraluduarte@uol.com.br 